\title{
Superharmonicity of nonlinear ground states
}

\section{Peter Lindqvist, Juan Manfredi and Eero Saksman}

Dedicated to the memory of Alberto P. Calderón (1921-1998)

\section{Introduction.}

The objective of our note is to prove that, at least for a convex domain, the ground state of the $p$-Laplacian operator

$$
\Delta_{p} u=\operatorname{div}\left(|\nabla u|^{p-2} \nabla u\right)
$$

is a superharmonic function, provided that $2 \leq p \leq \infty$. The ground state of $\Delta_{p}$ is the positive solution with boundary values zero of the equation

$$
\operatorname{div}\left(|\nabla u|^{p-2} \nabla u\right)+\lambda|u|^{p-2} u=0
$$

in the bounded domain $\Omega$ in the $n$-dimensional Euclidean space. Notice that for $p=2$ we have the ordinary Laplacian $\Delta=\Delta_{2}$, and in this case the inequality $\Delta u \leq 0$, expressing the superharmonicity, is evident from the equation $\Delta u+\lambda u=0$, since $\lambda>0$ and, by convention, $u>0$.

The underlying phenomenon is most clearly visible in the case $p=$ $\infty$, when our operator is to be understood as

$$
\Delta_{\infty} u=\sum_{i, j=1}^{n} \frac{\partial u}{\partial x_{i}} \frac{\partial u}{\partial x_{j}} \frac{\partial^{2} u}{\partial x_{i} \partial x_{j}}
$$


The superharmonicity is a consequence of two ingredients in the proof: i) $\Delta_{p} u \leq 0$ and ii) $\log u$ is concave in a convex domain. Our argument is based on the identity

$$
\Delta_{1} \log u+\frac{\Delta_{\infty} u}{|\nabla u|^{3}}=\frac{\Delta u}{|\nabla u|},
$$

from which we can read off that, if $\Delta_{1} \log u \leq 0$ and $\Delta_{\infty} u \leq 0$, then the desired inequality $\Delta u \leq 0$ holds. Unfortunately, the second derivatives needed to evaluate (1.4) do not always exist pointwise, making the identity difficult to use. The remedy is to interpret inequalities like $\Delta_{\infty} u \leq 0$ in the viscosity sense.

The important term $\Delta_{1} \log u$ calls for an explanation. The expression

$$
-\Delta_{1} v=-\operatorname{div} \frac{\nabla v}{|\nabla v|}
$$

is the mean curvature of the level surfaces of the function $v$. In the case of two independent variables this is the familiar expression

$$
k=-\frac{v_{y}^{2} v_{x x}-2 v_{x} v_{y} v_{x y}+v_{x}^{2} v_{y y}}{\left(v_{x}^{2}+v_{y}^{2}\right)^{3 / 2}}
$$

for the curvature of the level lines " $v(x, y)=$ constant". The operator ${ }^{1}$ $\Delta_{1}$ is "covariant"

$$
\Delta_{1} u=\Delta_{1} \log u
$$

The reason for passing to the logarithm is that, by Sakaguchi's extension [S] of the celebrated theorem of Brascamp and Lieb, $\log u$ is concave, where $u$ denotes the ground state of $\Delta_{p}$ in a convex domain. This has the effect that $\Delta_{1} \log u \leq 0$ in the viscosity sense.

Equations like $\Delta_{p} u=-2$ (the torsional creep problem) are also susceptible of our proof. In connexion with the $p$-harmonic capacitory function in convex rings similar phenomena have been detected by J. Lewis, cf. [Le]. See also [Ja, Lemma 2.4]. Our proofs do not work directly for $p \leq 2$, but we know that in the one-dimensional case the ground state is superharmonic for all $p \geq 1$. On the other hand the

1 As a matter of fact, the logarithm in (1.5) can be replaced by an arbitrary function. Instead of log-concave functions we may study so called quasi-concave functions. See [F]. 
assumption about convexity can be replaced by local convexity, except in Corollary 3.14.

The content is organized as follows. Viscosity supersolutions and the ground states are defined in Section 2. The main result is Theorem 3.12 and Corollary 3.14 in Section 3. Section 4 is a digression about concave functions and viscosity supersolutions. It can be read independently of the other sections.

\section{Some definitions.}

The concept of viscosity (super)solutions will be defined in this section. For a general introduction to this topic we refer to [C] and $[\mathrm{CC}]$. However, we begin with distributional solutions.

We assume that $\Omega$ is a bounded domain in the Euclidean $n$-dimensional space. The problem of minimizing the Rayleigh quotient

$$
\lambda_{p}=\min _{u} \frac{\int_{\Omega}|\nabla u(x)|^{p} d x}{\int_{\Omega}|u(x)|^{p} d x}
$$

among all functions $u$ in the Sobolev space $W_{0}^{1, p}(\Omega)$ has the EulerLagrange equation (1.2), when $1 \leq p<\infty$. (The right limit equation as $p \longrightarrow \infty$ is given in [JLM].) This is usually interpreted in the distributional sense, especiall y, when it comes to questions about existence and regularity.

Definition 2.2. We say that $u \in C(\Omega) \cap W_{0}^{1, p}(\Omega)$ is an eigenfunction of $\Delta_{p}$, if

$$
\int_{\Omega}|\nabla u|^{p-2} \nabla u \cdot \nabla \varphi d x=\lambda \int_{\Omega}|u|^{p-2} u \varphi d x
$$

for all $\varphi \in C_{0}^{\infty}(\Omega)$. Here $1<p<\infty$.

The ground state is the eigenfunction corresponding to the smallest possible value of $\lambda$, viz. the above mentioned minimum $\lambda_{p}$ of the Rayleigh quotient. The ground state $u_{p}$ exists and the eigenvalue $\lambda_{p}$ is simple. It does not change sign in $\Omega$ and our convention is that $u_{p}>0$. On the other hand, an eigenfunction that does not change sign must 
be a ground state. For all this we refer to $[\mathrm{Li}]$ and the references given there.

The case $p=\infty$ is more intricate. The ground state satisfies the equation

$$
\max \left\{\Lambda_{\infty}-|\nabla \log u(x)|, \Delta_{\infty} u(x)\right\}=0,
$$

in the viscosity sense (see the definition below). At each point, take the larger of the quantities. The eigenvalue

$$
\Lambda_{\infty}=\lim _{p \rightarrow \infty} \sqrt[p]{\lambda_{p}}
$$

is the radius of the largest ball that can be inscribed in $\Omega$. The variational ground state $u_{\infty}$ of $\Delta_{\infty}$ is obtained as a limit of $u_{p}$ 's as $p \longrightarrow \infty$. Unfortunately, the question of uniqueness has not been settled for $p=\infty$. We refer to [JLM] for a detailed discussion.

Definition 2.2 is not suitable to us. It is crucial that we can work with inequalities interpreted in the viscosity sense.

Definition 2.4. Suppose that $2 \leq p \leq \infty$. Let $u \in C(\Omega)$. We say that $\Delta_{p} u \leq 0$ in $\Omega$ in the viscosity sense, if at each given point $x \in \Omega$ we have $\Delta_{p} \varphi(x) \leq 0$ for all test-functions $\varphi$ touching $u$ from below at $x$. That is, $\varphi \in C^{\infty}(\Omega), \varphi(x)=u(x)$, and $\varphi(y)<u(y)$ when $y \neq x$.

A synonymous expression is that $u$ is a viscosity supersolution to the equation $\Delta_{p} u=0$. Notice that $\Delta_{2} u \leq 0$ in the viscosity sense exactly when $u$ is a continuous superharmonic function. ${ }^{2}$ (The definition, when written for lower semi-continuous functions, characterizes the whole class of superharmonic functions in the case $p=2$.) The family of test-functions depends on the point $x$.

Lemma 2.5. Suppose that $2 \leq p \leq \infty$. The ground state $u_{p}$ is a viscosity supersolution to the equation $\Delta_{p} u=0$.

Proof. The proof of this simple fact has been written out in [JLM, Lemma 1.8].

2 As a mnemonic rule, recall that a superharmonic function can be approximated from below by smooth functions and, accordingly, the test-function should touch from below. 


\section{Superharmonicity and concavity.}

Our aim is to prove the superharmonicity of the ground state of $\Delta_{p}, 2 \leq p \leq \infty$. The case $p=\infty$ will be based on the identity

$$
|\nabla \varphi|^{3} \Delta_{1} \varphi+\Delta_{\infty} \varphi=|\nabla \varphi|^{2} \Delta_{2} \varphi
$$

and the cases $2<p<\infty$ on the identity

$$
(p-2)|\nabla \varphi|^{3} \Delta_{1} \varphi+\frac{\Delta_{p} \varphi}{|\nabla \varphi|^{p-4}}=(p-1)|\nabla \varphi|^{2} \Delta_{2} \varphi
$$

both valid for smooth functions. At points where $\nabla \varphi=0$ we interpret the expressions so that there is no actual division by zero, for example

$$
\frac{\Delta_{p} \varphi}{|\nabla \varphi|^{p-4}}=|\nabla \varphi|^{2} \Delta \varphi+(p-2) \Delta_{\infty} \varphi
$$

We want to establish that $\Delta_{1} \log u \leq 0$, when $\log u$ is concave. This has to be done in the viscosity sense. Recall (1.5).

Lemma 3.3. Suppose that $\Omega$ is a convex domain. Let $u \in C(\Omega)$. If $u>0$ and $\log u$ is concave in $\Omega$, then

$$
|\nabla u|^{3} \Delta_{1} u \leq 0
$$

in the viscosity sense. That is, the inequality holds for positive testfunctions touching $u$ from below.

Proof. Fix a point $x \in \Omega$. Let $\varphi>0$ be any test-function touching $u$ from below at $x$. We have to prove that the expression

$$
|\nabla \varphi|^{3} \Delta_{1} \varphi=|\nabla \varphi|^{2} \Delta \varphi-\Delta_{\infty} \varphi
$$

is less or equal than 0 at the given point $x$. Now the function $\psi=\log \varphi$ will do as test-function at $x$ for the concave function $v=\log u$. Because

$$
|\nabla \varphi|^{3} \Delta_{1} \varphi=\varphi^{3}|\nabla \psi|^{3} \Delta_{1} \psi
$$

our claim is

$$
|\nabla \psi(x)|^{3} \Delta_{1} \psi(x) \leq 0 .
$$


To this end, observe that $\psi$ must be "concave at the point $x$ ", i.e.,

$$
\left\langle\xi, \mathcal{D}^{2} \psi(x) \xi\right\rangle \leq 0
$$

for all vectors $\xi \in \mathbb{R}^{n}$. (See Proposition 4.1 for the notation). Let $A=\mathcal{D}^{2} \psi(x)$. The matrix $A$ is negative semi-definite and symmetric. Hence its eigenvalues $\lambda_{1}, \lambda_{2}, \ldots, \lambda_{n}$ are negative or zero. The inequality

$$
|\nabla \psi(x)|^{2} \Delta \psi(x)-\Delta_{\infty} \psi(x) \leq 0
$$

which is our claim (3.6), can be written in the form

$$
|\xi|^{2} \operatorname{Trace}(A) \leq\langle\xi, A \xi\rangle,
$$

where $\xi=\nabla \psi(x)$. Diagonalizing the symmetric matrix $A$ as

$$
A=U \Lambda U^{-1}, \quad \Lambda=\operatorname{diag}\left[\lambda_{1}, \lambda_{2}, \ldots, \lambda_{n}\right],
$$

where $U$ is a unitary matrix, and denoting $\zeta=U^{-1} \xi$ we can write (3.8) as

$$
|\zeta|^{2}\left(\lambda_{1}+\lambda_{2}+\cdots+\lambda_{n}\right) \leq \lambda_{1} \zeta_{1}^{2}+\lambda_{2} \zeta_{2}^{2}+\cdots+\lambda_{n} \zeta_{n}^{2}
$$

This inequality is obviously true, because $\lambda_{1} \leq 0, \lambda_{2} \leq 0, \ldots, \lambda_{n} \leq 0$ and $|\zeta|^{2} \geq \zeta_{j}^{2}$. This proves (3.6).

At this stage we had better formulate an auxiliary result about the right-hand side in (3.1) and (3.2).

Lemma 3.9. Suppose that $\Omega$ is a convex domain. If $u>0, \log u$ is concave, and

$$
|\nabla u|^{2} \Delta u \leq 0
$$

in $\Omega$ in the viscosity sense, then $u$ is superharmonic in $\Omega$.

Proof. Fix $x \in \Omega$ and let $\varphi$ denote a positive test-function touching $u$ from below at $x$. By the assumption

$$
|\nabla \varphi(x)|^{2} \Delta \varphi(x) \leq 0
$$

We claim that $\Delta \varphi(x) \leq 0$. This is clear, if $\nabla \varphi(x) \neq 0$. 
If $\nabla \varphi(x)=0$, then a simple computation yields

$$
\varphi(x) \Delta \log \varphi(x)=\Delta \varphi(x)
$$

and hence our claim is that $\Delta \log \varphi(x) \leq 0$ in this case. The function $\log \varphi$ touches $\log u$ from below at $x$. Since $\log u$ is concave, $\log \varphi$ must be "concave at $x$ " and hence $\Delta \log \varphi(x) \leq 0$. (See Proposition 4.1.)

Thus $\Delta \varphi(x) \leq 0$ in both cases. Because $u(x)>0$, the restriction that $\varphi$ be positive has no influence on our conclusion that $\Delta u \leq 0$ in the viscosity sense. Functions that are superharmonic in the viscosity sense are superharmonic (in the ordinary sense).

Our main result is the following.

Theorem 3.12. Let $\Omega$ be a convex domain and suppose that $u \in C(\Omega)$ satisfies:

i) $u>0$ and $\log u$ is concave

ii) $\Delta_{p} u \leq 0$ in the viscosity sense for some $p, 2 \leq p \leq \infty$,

in $\Omega$. Then $u$ is superharmonic in $\Omega$.

Proof. Fix a point $x \in \Omega$ and let $\varphi$ be a positive test-function touching $u$ from below at $x$. In the case $p=\infty$ we use Equation (3.1). According to Lemma 3.3 the first term is less or equal than 0 and so is the second term according to ii). Thus

$$
|\nabla \varphi(x)|^{2} \Delta \varphi(x) \leq 0
$$

The desired superharmonicity follows from Lemma 3.9. This was the case $p=\infty$. - The cases $2<p<\infty$ are based on Equation (3.2), but otherwise similar.

REMARK. A little more can be proved. If $\log u$ is concave and if $\Delta_{p} u \leq$ 0 for some $p \geq 2$, then $\Delta_{q} u \leq 0$ for all $q$ in the range $2 \leq q \leq p$, the inequalities being interpreted in the viscosity sense. To see this, use the identity

$$
(p-2) \frac{\Delta_{q} \varphi}{|\nabla \varphi|^{q-4}}=(p-q)|\nabla \varphi|^{2} \Delta \varphi+(q-2) \frac{\Delta_{p} \varphi}{|\nabla \varphi|^{p-4}}
$$


Corollary 3.14. In a convex bounded domain $\Omega$ the ground state of the operator $\Delta_{p}$ is a superharmonic function, provided that $2 \leq p<\infty$. The same concerns any variational ground state of $\Delta_{\infty}$.

Proof. By [S, Theorem 1] $\log u$ is concave and by Lemma $2.5 \Delta_{p} u \leq 0$ in the viscosity sense. The result follows from Theorem 3.12.

As we indicated in the Introduction, the solution of the "torsional creep equation" $\Delta_{p} u=-2,2 \leq p \leq \infty$ is superharmonic in a convex domain. Indeed, if the solution $u$ has boundary values zero, then the function $u^{1-1 / p}$ is concave a ccording to [S, Theorem 2]. Thus condition i) holds a fortiori. Condition ii) has been established in [BDM]. - Needless to say, there are many other interesting situations where Theorem 3.12 applies.

\section{About Concave Functions.}

It is well-known that the negative semi-definiteness of the Hessian matrix characterizes concave functions with continuous second partial derivatives. Interpreted in the viscosity sense this characterizes all (locally) concave functions. This is likely* to be known to the experts in the field.

Proposition 4.1. Let $u \in C(\Omega)$, where $\Omega$ is a convex domain. Then the function $u$ is concave in $\Omega$ if and only if

$$
\left\langle\xi, \mathcal{D}^{2} u \xi\right\rangle=\sum_{i, j=1}^{n} \frac{\partial^{2} u}{\partial x_{i} \partial x_{j}} \xi_{i} \xi_{j} \leq 0
$$

in the viscosity sense for all $\xi \in \mathbb{R}^{n}$. That is, whenever $x \in \Omega$ and $\varphi \in C^{2}(\Omega)$ are such that

i) $\varphi(x)=u(x)$,

ii) $\varphi(y)<u(y)$, when $y \neq x$,

then

$$
\left\langle\xi, \mathcal{D}^{2} \varphi(x) \xi\right\rangle \leq 0
$$

\footnotetext{
* Added in proof: See "Hessian Measures II", Annals of Mathematics (to appear),
} by N. Trudinger and X.-J. Wang, for reaching extensions of Proposition 4.1. 
for all $\xi \in \mathbb{R}^{n}$.

Notice that, as usual, each point in $\Omega$ requires its own family of test-functions $\varphi$ touching from below. If (4.3) holds for all such $\varphi$, it also holds for all $\varphi$ with ii) weakened to $\varphi \leq u$.

Proof of the Proposition. Assume that $u$ is concave. Fix a vector $\xi \neq 0$ and let $x$ be a point in $\Omega$. Let $\varphi$ be any test-function touching $u$ from below at $x$. Then $\varphi$ must be "concave at $x$ ", that is

$$
\frac{d^{2} \varphi(x+t \xi)}{d t^{2}} \leq 0, \quad \text { at } t=0
$$

since otherwise i) and ii) would contradict the concavity of $u$ itself. After differentiation, this inequality is exactly (4.3) and so we have proved the first half of the proposition.

For the other direction of the proof, we assume that $u$ is not concave. We may assume that the ball $|x| \leq 2$ is comprised in $\Omega$ and, by adding a linear function and scaling, that

$$
u( \pm 1,0, \ldots, 0) \geq 2, \quad u(0,0, \ldots, 0)=0 .
$$

There is a small $\sigma>0$ such that $u(x)>1$, when $|x|=1$ and $x_{2}^{2}+\cdots+$ $x_{n}^{2}<\sigma^{2}$.

We will construct a test-function of the form

$$
\varphi(x)=a+\varepsilon x_{1}^{2}-\frac{x_{2}^{2}+\cdots+x_{n}^{2}}{\varepsilon}
$$

touching $u$ from below at some point $x$ with $|x|<1$. The touching point is to be determined later! We assume that $a \leq 0$ and $0<\varepsilon<1$. Then

$$
\varphi(0)=a \leq 0=u(0) .
$$

Let

$$
m=\min _{|x| \leq 1} u(x)
$$

and fix $\varepsilon$ so that

$$
0<\varepsilon<\frac{\sigma^{2}}{1-m},
$$

notice that $m \leq 0$. We claim that

$$
\varphi(x)<u(x), \quad \text { when }|x|=1 .
$$


This is independent of $a \leq 0$. To see this, consider first the points where $u(x)>1$. Always

$$
\varphi(x) \leq a+\varepsilon \cdot 1 \leq 1
$$

and so we have only to check the points where $|x|=1$ and $x_{2}^{2}+\cdots+x_{n}^{2}>$ $\sigma^{2}$. There

$$
\varphi(x) \leq a+\varepsilon-\frac{\sigma^{2}}{\varepsilon}<1-\frac{\sigma^{2}}{\varepsilon} \leq m \leq u(x) .
$$

Thus (4.5) is verified.

If $a$ is negative enough, $\varphi(x)<u(x)$ when $|x| \leq 1$. Select the largest $a$ such that $\varphi(x) \leq u(x)$, when $|x| \leq 1$. The corresponding $\varphi$ must touch $u$ at some point $x$ with $|x|<1$, since $\varphi(x)<u(x)$, when $|x|=1$ for all $a \leq 0$. At this point $\varphi$ will do as test-function. However, the indefinite quadratic form

$$
\left\langle\xi, \mathcal{D}^{2} \varphi(x) \xi\right\rangle=2 \varepsilon \xi_{1}^{2}-\frac{2}{\varepsilon}\left(\xi_{2}^{2}+\cdots+\xi_{n}^{2}\right)
$$

violates (4.3). This concludes our proof.

In passing, we mention that, usually, classical solutions are viscosity solutions, but this is not the case for the Monge-Ampère equation

$$
u_{x x} u_{y y}-u_{x y}^{2}=0
$$

in two variables. A plain example is

$$
u(x, y)=\cos y \text {. }
$$

It definitely satisfies the equation, though not in the viscosity sense. The following curious fact seems to have passed unnotized in the literature.

Proposition 4.6. Let $u \in C(\Omega)$, where $\Omega$ is a convex domain in $\mathbb{R}^{2}$. Then $u$ is concave if and only if

$$
u_{x y}^{2}-u_{x x} u_{x y} \leq 0
$$

in the viscosity sense, the test-functions touching $u$ from below. 
We skip the proof, because this is far off from our central theme. It can be based on Proposition 4.1. A more direct construction is to determine the touching point of the test-function as in the proof of Proposition 4.1.

Acknowledgement. We thank the anonymous referee for some helpful suggestions.

\section{References.}

[A] Aronsson, G., Extension of functions satisfying Lipschitz conditions. Arkiv för Matematik 6 (1967), 551-561.

[BDM] Bhattacharya, T., Di Benedetto, E., Manfredi, J., Limits as $p \longrightarrow \infty$ of $\Delta_{p} u_{p}=f$ and related extremal problems. Classe Sc. Math. Fis. Natl. Rendiconti del Seminario Matematico. Fascicolo Speciale Non Linear PDE's, Universita'e Poli tecnico Torino, (1989), 15-68.

[BL] Brascamp, H., Lieb, E., On extension of the Brunn-Minkowski and Prékopa-Leindler theorems, including inequalities for log concave functions, and with an application to the diffusion equation. J. Funct. Anal. 22 (1976), 366-389.

[CC] Caffarelli, L., Cabré, X., Fully Nonlinear Elliptic Equations. Colloquium Publications 43, American Mathematical Society, 1995.

[C] Crandall, M., Viscosity solutions: A primer. Lecture Notes in Math. 1660, Springer, 1997, 1-43.

[F] Fenchel, W., Über konvexe Funktionen mit vorgeschriebenen Niveaumannigfaltigkeiten. Math. Z. 63 (1956), 496-506.

[Ja] Janfalk, U., Behaviour in the limit, as $p \longrightarrow \infty$, of minimizers of functionals involving $p$-Dirichlet integrals. SIAM J. Math. Anal. 27 (1996), 341-360.

[Je] Jensen, R., Uniqueness of Lipschitz extensions - Minimizing the sup norm of the gradient. Arch. Rational Mech. Anal. 123 (1993), 51-74.

[JLM] Juutinen, P., Lindqvist, P., Manfredi, J., The $\infty$-eigenvalue problem. Arch. Rational Mech. Anal. 148 (1999), 89-105.

[Le] Lewis, J., Capacitary functions in convex rings. Arch. Rational Mech. Anal. 66 (1997), 201-224.

[Li] Lindqvist, P., On non-linear Rayleigh quotients. Potential Anal. 2 (1993), 199-218. 
[S] Sakaguchi, S., Concavity properties of solutions to some degenerate quasilinear elliptic Dirichlet problems. Annali della Scuola Normale Superiore de Pisa, Serie IV (Classe di Scienze) 14 (1987), 403-421.

Recibido: 4 de septiembre de 1.998

Peter Lindqvist

Department of Mathematical Sciences Norwegian University of Science and Technology

N-7034 Trondheim, NORWAY

lqvist@math.ntnu.no

Juan Manfredi

Department of Mathematics

University of Pittsburgh

Pittsburgh, PA 15260, U.S.A.

manfredi+@pitt.edu

and

Eero Saksman

Department of Mathematics

P.O. Box 4

FIN-00014 University of Helsinki, FINLAND

saksman@cc.helsinki.fi 